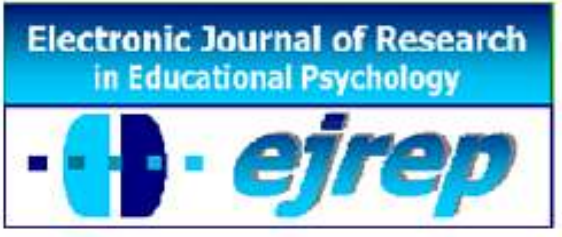

\title{
La Clave Profesional: Validación de un Instrumento de Orientación Vocacional
}

\section{María J. Mudarra ${ }^{1}$ y Ángel Lázaro Martínez ${ }^{2}$}

${ }^{1}$ Vicedecana de Estudiantes y Educación Social. Departamento de Métodos de Investigación y Diagnóstico en Educación (Orientación Educativa, Diagnóstico e Intervención Psicopedagógica) (MIDE II-OEDIP), UNED

${ }^{2}$ Catedrático Emérito. Área MIDE. Universidad de Alcalá

\section{España}

Correspondencia: María José Mudarra. Dpto. MIDE II. Facultad de Educación. UNED. C/ Juan del Rosal 14. 28040. Madrid. Spain. E-mail: mjmudarra@edu.uned.es 


\section{Resumen}

Introducción. La Clave Profesional es un instrumento de diagnóstico para la Orientación Vocacional inspirado en "The Career Key" de Lawrence Jones, basado en el modelo RIASEC de Holland. En un contexto de reflexión sobre la identificación personal con una profesión, con las formas de vida que orientan los valores y los tipos de personalidad, se estudia la validez cultural y las propiedades psicométricas de la Clave Profesional en una muestra española.

Método. La muestra española estuvo compuesta por 517 estudiantes de Educación Secundaria de diferentes niveles educativos (edades comprendidas entre 15 y 18 años) equilibrada respecto al género y al tipo de centro educativo (público, privado). Los instrumentos aplicados fueron, además de La Clave Profesional, la Investigación Autodirigida -SDS de Holland- las Formas de Vida de Spranger y el 16 PF de Cattell. Además de análisis correlacionales entre constructos, se calcularon índices de fiabilidad y validez (alpha de Cronbach, KR-20, índices de homogeneidad y validez de los ítems) así como análisis factoriales sobre las secciones de la Clave Profesional.

Resultados. Los índices de discriminación y consistencia interna demuestran la elevada fiabilidad de la Clave Profesional. Paralelamente se verifica su adecuada validez (criterial y de constructo). Además, se demuestra la existencia de relaciones significativas entre los constructos de intereses, estilos vitales -valores- y personalidad.

Discusión y conclusiones. Los resultados de fiabilidad y validez obtenidos demuestran que la Clave Profesional posee una calidad científico-técnica adecuada para ser aplicada a la población española. Además, las relaciones halladas entre las escalas RIASEC, los factores de personalidad y los estilos vitales sugieren que es preciso seguir investigando particularmente algunos tipos como el emprendedor, el neurótico y el extrovertido.

Palabras clave: Modelo RIASEC de Holland, la Clave Profesional, Orientación Vocacional, Intereses Profesionales, estudio de validación. 


\title{
La Clave Profesional: Validation of a Vocational Guidance Instrument
}

\begin{abstract}
Introduction. The Career Key: Validating a tool for Career Assessment and Guidance. The current study demostrates empirical and cultural validity of La Clave Profesional (spanish adaptation of Career Key, Jones's test based on Holland's RIASEC model). The process of providing validity evidences also contains a reflection on personal and career development and examines the relationships between RIASEC types, personality traits and Spranger`s Life Style.
\end{abstract}

Method. The participants in this study were 517 secondary students aged between 15 and 18 years of public and private high schools. Career, lifestyle and personality factors were assessed by Career Key, Holland's Self-Directed Search, Spranger Lifestyle Test and Cattell' $16 \mathrm{PF}$.

Results. The discrimination index of the items and internal consistency of scales have effectively shown that La Clave Profesional is a very reliable instrument. Additionally, validity procedures demostrate its relevant validity (criterial and construct). Furthermore, significant relationships were found between interest and personality factors.

Discussion and conclusions. Results reveal that La Clave Profesional has sufficient scientific and technical credibility for application to the Spanish population. Once again, relationships between RIASEC scales, personality traits and lifestyle choices suggests more research about enterprising, neurotic and extraversion types.

Keywords: Holland`s RIASEC model, Career Key, Career Guidance, vocational interest, validation study 


\section{Introducción}

El propósito de este artículo es presentar el estudio empírico de validación de la Clave Profesional, un instrumento diagnóstico para la Orientación Vocacional inspirado en el Career Key (Jones, 1987) en un contexto de reflexión sobre la necesidad de diagnosticar estilos vitales y orientar elecciones profesionales propias de una sociedad compleja, contradictoria en sus planteamientos y constantemente cambiante.

\section{La personal forma de ser en el entramado ambiental a través de las elecciones profesionales}

En la cultura occidental actual, caracterizada por la multiplicidad de relaciones y enfoques, se tiene la posibilidad de elegir porque el ambiente social sigue siendo diverso, disperso y difuso, tal como se definió hace años (García-Yagüe y Lázaro, 1971). Podríamos añadir que tal ambiente social tiende a ser manipulable, en ocasiones, tremendamente fatalista y, desde luego, capaz de invadir la intimidad de la persona, su capacidad de reflexión y sus opciones de elección vital. Si la capacidad de elección es un rasgo intrínseco al proceso de decisión, una elección profesional responsable reclama la adquisición de ciertas competencias y la adaptabilidad de la persona a su entorno próximo de modo que pueda decidir con coherencia personal y social.

Toda persona despliega, desde un núcleo de posibilidades inicialmente dadas por la herencia y por el entramado ambiental de su fermento emocional, la amplitud de su propio itinerario el cuál se va construyendo, no como consecuencia fatal del destino, sino a través de sus decisiones personales, con implicaciones consecuentes y responsables. Particularmente, desde mediados de los años noventa se vienen realizando diversos estudios con el propósito de proporcionar recursos que mejoren el nivel de información ocupacional al tiempo que promuevan la adquisición de una estructura cognitiva adecuada que favorezca el desarrollo del itinerario personal y profesional. Se supone que la identificación personal con una profesión es una constante del desarrollo necesaria para lograr la integración satisfactoria y productiva de la personalidad. Basándonos en el enfoque del fortalecimiento (empowerment), en este estudio se analiza la identificación con las formas de vida que orientan los valores y los tipos de personalidad. Ampliando el concepto biológico de tropismo, puede considerarse que los tropismos de personalidad son tendencias que se formalizan en el ser de cada uno, en una manera concreta de existir conforme a valores asumidos, manifiestos en actitudes y desarrollados según los estímulos de un medio ambiente próximo. Así, una forma de ser (tipo 
de personalidad) es un travesía que se empeña constantemente en surcar una superficie ondulatoria, irregular y aparentemente caótica, como la superficie de las aguas, en donde el navegante pilota su vida, su itinerario, esperando mejorar en cada singladura, hasta que alcanza el punto de llegada. La forma de navegar y el tipo de navegación están condicionadas por el ámbito en el que se navega y lo que se quiere patronear; configurando la personal forma de ser de cada uno que se concreta en el desarrollo de su recorrido y se decide en cada momento.

La autoevaluación profesional: un proceso clave en la Orientación para el Desarrollo de la Carrera

La Orientación para el Desarrollo de la Carrera enfatiza tal carácter dinámico del desarrollo personal como proceso evolutivo en el que se integran sucesivas elecciones vocacionales, promoviendo adecuados procesos de decisión en todos los ámbitos en que se desenvuelve el individuo (educativo, laboral, ocio y tiempo libre). Tales intervenciones orientadoras, comprensivas, sistemáticas y planificadas, se deben fundamentar en un proceso previo de diagnóstico y evaluación profesional (Career Assessment).

La autoevaluación profesional es un proceso que permite a la persona ir descubriéndose paulatinamente, revisar sus planteamientos y evolución personal según las experiencias asumidas y las variaciones del ambiente, las pretensiones personales y las oportunidades sociales. Exige recoger toda información relevante que permita optimizar el proceso de decisión profesional al tiempo que favorecer aprendizajes, identificar problemas, sugerir alternativas y estimular acciones, cualidades cada vez más presentes en los instrumentos diagnósticos actuales, especialmente si son autoaplicables. Muchos de estos instrumentos se integran en programas de Orientación Profesional como "Tengo que decidirme” (Álvarez Rojo, 1991), “Toma la iniciativa” (Delgado, 1995), “De Gira hacia el Trabajo" (Romero, 1997) o Programa de Orientación Profesional Autoaplicado POPA (Corominas, Álvarez y Bisquerra, 1999), “Tu futuro profesional” Repetto (1999) "Sistema de Autoayuda Vocacional” (Rivas y López, 2003); o más recientemnte “GR: Herramienta de autoaplicación para la orientación académica y profesional" (Corominas et al 2009) o el "Programa de Orientación Educativa y Sociolaboral POES (Santana Vega et al. 2009)

Precisamente como elemento estimulador, que ayude a trazar perfiles en los momentos iniciales de las decisiones personales, se ha elaborado y validado un instrumento diagnóstico 
denominado Clave Profesional. Basado en diversos modelos teóricos -tipologías de formas de ser, personalidad, intereses, elecciones vocacionales- particularmente inspirado en planteamientos de Spranger (1935), Holland (1959) y Jones (1987). Permite localizar los estilos y formas de vida predominantes de modo que la persona pueda ajustar sus características a las posibilidades y contextos ambientales de forma semejante a la Investigación Autodirigida o Self-Directed Search (SDS, de Holland, 1979, 1994).

\section{El Modelo de Holland}

Holland (1997) identificaba la integración de la personalidad con el desarrollo de Tipos de Personalidad, definiendo la madurez vocacional en función de la madurez que manifiesta un individuo al realizar elecciones vocacionales congruentes según ciertos tipos de personalidad y ambiente. Aunque su enfoque parezca desmesurado y algunos de sus constructos teóricos sean ampliamente cuestionados (Tracey \& Robbins, 2006), sus propuestas han tenido enorme impacto científico (Gottfredson, 1999). Esta popularidad puede explicarse por las características más apreciadas de sus instrumentos: autoaplicabilidad, sencillez en la formulación y transparencia en sus escalas, evidencias empíricas de su influencia relativas a la autocomprensión de la conducta vocacional y a la flexibilidad para establecer nuevas relaciones conceptuales (aprendizaje de toma de decisiones, organización de la información ocupacional o la estructuración de programas educativos de desarrollo de carrera). Así, el Modelo Tipológico Hexagonal de Intereses y Personalidad o, más brevemente, Modelo RIASEC, se ha depurado teóricamente en numerosas ocasiones (Holland, 1962, 1968, 1985, 1997; Gottfredson \& Holland, 1991; Holland \& Gottfredson, 1976, 1978; Holland \& Nichols, 1964; Holland, Sorensen, Clark, Nafzinger \& Blum, 1973), para incidir más tanto en el proceso de adaptación del individuo a contextos cambiantes (en función de sus características personales, conocimiento ocupacional y capacidad de transferencia informativa) como en el estudio específico de los problemas que surgen a lo largo de dicho proceso, cuando el sistema de adaptación del individuo resulta rudimentario o falla, provocando decisiones profesionales que generan inseguridad o insatisfacción personal.

El resultado de la combinación específica entre intereses y aptitudes genera cierta disposición en la persona a pensar, percibir y actuar de forma particular (Holland, 1997). Cada uno de estos estilos vitales pueden referirse a los tipos de personalidad definidos por Holland: Realista (R), Investigador (I), Artístico (A), Social (S), Emprendedor (E) y Convencional (C) a los cuáles les corresponden seis tipos de ambientes paralelos. Según el grado de adaptación 
del individuo al entorno -coherencia entre tipos personales y ambientales- se pueden pronosticar aspectos como la elección profesional, el logro educativo, competencia personal, conducta social y susceptibilidad a la influencia ambiental (Martínez Vicente y Valls, 1999), estabilidad y satisfacción profesional (Porter \& Umbach, 2006), la persistencia en la elección profesional (Allen \& Robbins, 2008), la estabilidad en los resultados académicos en función del ambiente (Smart, 2010) e incluso el estatus profesional estimado según el salario (KristofBrown, Zimmerman \& Johnson, 2005).

Para diagnosticar el perfil de tipos característico de un individuo, Holland propuso diversos instrumentos e indicadores de madurez vocacional (consistencia, congruencia, diferenciación) basados en las relaciones entre tipos, acordes a su modelo, esto es, las relaciones o semejanzas entre los tipos son inversamente proporcionales a las distancias entre ellos en el hexágono. Tal como señala Savickas y Gottfredson (1999), uno de los últimos constructos incorporados al modelo, la identidad vocacional, se refiere tanto a la estabilidad de las metas y autopercepciones personales como a la claridad de las metas explícitas o expectativas ambientales.

Formas de Vida de Spranger: otro enfoque de aproximación a la validez del Modelo RIASEC

En la validación de la Clave Profesional, además se han considerado los estudios de Eduard Spranger (1935), autor relegado en el paradigma actual del desarrollo de carrera, a pesar de su relevancia, pues propuso un instrumento de medida semejante al que posteriormente elaborara Holland, pero con un asentamiento teórico -desde nuestro punto de vista- más sólido, flexible y amplio. A Spranger se le catalogó antes de la década de los cincuenta, como un psicólogo científico-espiritual, además de un filósofo de la vida, pues él deseaba comprender la personalidad, descubrir el sentido de las formas en las que se manifiesta el espíritu objetivo, que se concretan en el esfuerzo y en la lucha de la individualidad por consolidar el sentido de lo humano. Esta comprensión se refiere a los valores que fundamentan la realidad; lo comprendido tiene un sentido, un valor, una dirección y un lugar en la estructura total de la personalidad, cuya expresión máxima es un sistema global, personal de valores objetivos. Spranger (1935) distingue los siguientes tipos o formas de vida: (T) Teórico, (E) Económico, (S) Social, (A) Estético, (P) Político y (R) Religioso. Estos tipos se localizan a través de las preferencias situacionales y las predisposiciones que manifiestan los valores consolidados en cada persona. Este planteamiento, desde perspectivas diferentes, lo desarrollaron también autores como Allport y Vernon (Allport y Vernon, 1931; 
Allport, Vernon y Lindzey, G., 1951), quiénes difundieron una teoría de la personalidad que alcanzó gran difusión y popularidad, generando un instrumento de medida denominado "Formas de vida", publicada por el Instituto de Pedagogía "San José de Calasanz" del Consejo Superior de Investigaciones Científicas, cuya traducción y adaptación española realizó García-Yagüe (1957).

Ahora bien, es necesario analizar la validez cultural de estos modelos teóricos. La estructura de los intereses profesionales y su relación con otros modelos de personalidad, han generado multitud de debates científicos e investigaciones en distintos países con muestras culturalmente diversas para verificar el cumplimiento de sus principios (Gottfredson, 1999). En la población española, respecto al modelo de Holland, destacan las investigaciones de Castaño, López Navarro y Domínguez (1979) con una muestra de estudiantes universitarios; Fernández Eire (1997) con adolescentes gallegos; Martínez Vicente y Valls, (2001, 2006) con una muestra de individuos de 12 a 41 años, Martínez-Vicente (2001, 2007) y Mudarra (2003, 2007) con una muestra de 762 alumnos de Educación Secundaria.

Utilizando el SDS como instrumento de referencia común en las citadas investigaciones, estos autores concluyen que la estructura de personalidad propuesta por Holland se manifiesta en las muestras estudiadas, siendo especialmente fiables las escalas Social y Convencional, (Lewis \& Rivkin, 2000; Mudarra, 2007; Rounds et al., 1999), si bien surgen algunos matices, como la localización de una mayor afinidad entre los tipos indicados en el caso de los adolescentes varones, (Fernández Eire, 1997); la constatación de la presencia de pequeños desajustes entre los tipos que se concentran en la relación RC y en la relación del tipo I con los tipos A, S y C, (Valls Fernández y Martínez Vicente, 2001). También se constatan las preferencias de las mujeres por los intereses sociales y artísticos a diferencia de los varones que prefieren los realistas (Mudarra, 2003). Probablemente este resultado pueda explicarse por los roles culturales tradicionales y las expectativas de autoeficacia asociadas a los mismos pero, en todo caso, requiere una llamada de atención a los orientadores para evitar sesgos debidos al género tanto en los instrumentos como en las elecciones profesionales. Paralelamente, suelen confirmarse las relaciones entre los modelos de personalidad -como el Big Five- y de intereses, se obtienen relaciones moderadas a menudo moduladas por la variable género, (Caprara et al, 2001; Mudarra, 2003; Tokar \& Swanson, 1995). Así, parece que los diferentes tipos tienden a ser estables durante cierto período 
cronológico, pero sufren cambios -incluso radicales- en otros períodos de la vida, dependiendo de los valores y circunstancias sociales y personales.

\section{Objetivos}

En definitiva, siguen siendo necesarios estudios para verificar la validez cultural del modelo tipológico de Holland y de los instrumentos basados en él. Desde una perspectiva empírica, el objetivo del estudio de validación planteado en este artículo es comprobar el modelo RIASEC en una muestra española. Paralelamente, se analizan algunos de los supuestos teóricos como las relaciones de estos tipos con los valores y los factores de personalidad. De este modo, además de enriquecer y clarificar los resultados de investigaciones anteriores, se aportan criterios para valorar la aplicación de un nuevo instrumento psicopedagógico, la Clave Profesional, al tiempo que se consolida su configuración teórica según las derivaciones del sistema y la jerarquía de valores propuestos por Spranger.

\section{Método}

\section{Participantes}

Los participantes de este estudio fueron 517 alumnos, procedentes de cuatro centros educativos de la Comunidad de Madrid, seleccionados intencionalmente conforme los siguientes criterios: equilibrio entre centros públicos y privados, homogeneidad respecto al nivel socio-económico-cultural (en este caso intermedio) y accesibilidad de la muestra conforme a los recursos disponibles en el estudio y el código deontológico. Así, se obtuvo una muestra equilibrada respecto al tipo de centro $(40 \%$ de alumnos de centros privados y $60 \%$ de Institutos de Enseñanza Secundaria. En particular, se seleccionaron alumnos de 15 a 18 años, es decir, pertenecientes a $1^{\circ}$ de Bachillerato LOGSE y a $2^{\circ}$ de Bachillerato LOGSE, por ser estas edades más estables en sus tendencias profesionales que en los primeros cursos de Educación Secundaria -además, las materias científicas y profesionales ya iniciadas les permiten descubrir sus actividades preferidas). Respecto al nivel educativo, se observa un porcentaje ligeramente superior de alumnos pertenecientes a $1^{\circ}$ de Bachillerato (un 57\%).

Si tenemos en cuenta el género de estos alumnos la muestra resulta equilibrada $(47,8 \%$ de varones y $52,2 \%$ de mujeres) con ligero predominio de las mujeres en ambos niveles 
educativos $\left(53,5 \%\right.$ frente a $46,5 \%$ en $1^{\circ}$ de Bachillerato y $50,5 \%$ frente a $49,5 \%$ en $2^{\circ}$ de Bachillerato) como sucede en investigaciones semejantes (Valls Fernández y Martínez, 1999).

\section{Instrumentos}

\section{La Clave Profesional (Lázaro y Mudarra, 2014)}

Es un instrumento autoaplicable inspirado en el Carrer Key de Jones (1987) quién, seguidor de las ideas de Holland, desarrolla sus propuestas en la Universidad de Carolina del Norte. Publicado por primera vez por Ferguson Publishing Company se convierte, a partir de 1997, en un instrumento aplicable también a través de internet (www.careerkey-ca.org), como un servicio público gratuito para estudiantes de Enseñanza Secundaria, adaptado para múltiples países (Canadá, México, India, China, Corea, Caribe, Rumanía, Vietnam, Turquía...). Ambas formas -electrónica y lápiz y papel- únicamente difieren en las posibilidades que internet ofrece como sistema interactivo en tiempo real (por ejemplo, la posibilidad de consultar en cualquier momento toda la información disponible sobre una determinada ocupación. Tal como señala su autor (Jones, 1989) es un instrumento muy parecido al SDS (Holland, 1994) en varios aspectos: la autoevaluación conforme según los 6 tipos de Holland, la identificación de nuevas ocupaciones en la Lista de ocupaciones que adjunta la misma prueba y la propuesta de pasos a realizar, como parte de un proceso de Orientación profesional que exige la exploración de la carrera y facilita la toma de decisiones.

La Clave Profesional que ofrecemos, semejante a las pruebas de Holland y Jones, se estructura en las siguientes secciones recogidas en la Figura 1.

\section{$\mathbf{1}^{\text {a }}$ sección, ¿Cómo me veo a mí mismo?}

Trata de valorar mediante una escala de 3 puntos el grado de verdad de 24 ítems descriptivos de la persona, agrupados en los 6 tipos de Holland, en torno a 4 núcleos temáticos.

\section{$2^{\mathrm{a}}$ sección, Ocupaciones atractivas}

Presenta 36 ocupaciones extraídas del Diccionario de Códigos Ocupacionales de Holland en grupos de 6 para estimar el grado de interés que despierta cada ocupación en una escala de 3 puntos.

\section{$3^{\text {a }}$ sección, Valoración global}


Recoge la puntuación global en cada tipo RIASEC como combinación lineal de las puntuaciones totales obtenidas en los 60 ítems de la prueba ( $1^{\text {a }}$ sección, 24 ítems; $2^{\mathrm{a}}$ sección, 36 ítems). La configuración tipológica característica del individuo, definida por los tipos en que obtuvo mayor puntuación, se representa gráficamente simulando una llave cuyos puntos son los picos del perfil.

$4^{\text {a }}$ sección, Listado de Ocupaciones

Presenta 371 ocupaciones clasificadas según el modelo RIASEC.

$5^{\text {a }}$ sección: Siguientes pasos a seguir

Ofrece orientaciones para favorecer el desarrollo de carrera.

Figura 1. Secciones de la Clave Profesional

\section{La Investigación Autodirigida (SDS) (Holland, 1994)}

Esta prueba, también autoaplicable, se compone del Cuaderno de Evaluación -con las secciones de Fantasías vocacionales, Preferencias, Competencias, Trabajos, Habilidades- el Descubridor de ocupaciones -1335 ocupaciones ordenadas según los tipos y el nivel educativo- y el Cuaderno interpretativo: usted y su carrera, dónde se ofrecen orientaciones sobre las interpretaciones, problemas frecuentes y fuentes adicionales de información. Aunque en este estudio se ha empleado la adaptación de Holland (1994), existen numerosas adaptaciones entre las que destacan las de Martínez-Vicente, (2001) y Martínez-Vicente y Valls, (2006).

Las Formas de vida de Spranger (Spranger, 1935)

Para estudiar la relación del modelo tipológico de Holland con los valores y los factores de personalidad se aplicaron dos instrumentos: el Cuestionario de Ideas Personales (García-Yagüe, 1957, Instituto de Pedagogía de San José de Calasanz, adaptación al castellano de las Formas de Vida de Spranger, 1935) y el Cuestionario Factorial de Personalidad 16 PF (Cattell, 1975). El Cuestionario de Ideas Personales señala 6 Formas de Vida o valores que se corresponden con 6 tipos ideales de hombres (teórico, económico, estético, social, político y espiritual) conforme el valor predominante en cada uno. Para explorar las Ideas Personales del alumno, además de expresar sus preferencias, debe emitir juicios sobre la importancia que tienen para él, ciertos enunciados -valores-. 
El Cuestionario Factorial de Personalidad (16 PF) (Cattell, 1975)

Ampliamente difundido, no requiere una descripción detallada, baste señalar su fundamentación teórica factorial, la adecuada fiabilidad y validez que presentan sus escalas.

\section{Procedimiento}

Para comprobar la estructura tipológica de Holland y validar la Clave Profesional, los investigadores principales con un equipo de colaboradores -asignados al azar a los distintos grupos de alumnos- aplicaron la Clave Profesional junto al SDS, el 16 PF y las Formas de Vida de Spranger, respetando tanto el orden de aplicación como el intervalo de tiempo entre aplicaciones para evitar sesgos debidos al efecto de maduración. En ningún caso la aplicación total llegó a superar los 90 minutos. Se realizaron diversos análisis de fiabilidad y validez para estudiar las características técnicas de la Clave Profesional y otros estudios complementarios correlacionales - con el 16 PF y las Formas de Vida- para explorar su validez de constructo y proponer decisiones de mejora.

\section{Análisis de datos}

El análisis de datos se llevó a cabo utilizando el programa IBM-SPSS 19. Para determinar la consistencia interna de cada escala RIASEC y la fiabilidad global de la prueba se calculó el estadístico $\alpha$ de Cronbach, la validez criterial y la validez concurrente a través de los índices de asociación entre sus escalas y las de la Investigación Autodirigida -SDS-. La estructura hexagonal de la Clave Profesional se exploró a través de diversos análisis factoriales, utilizando el método de Componentes Principales. El funcionamiento de los ítems dentro de sus escalas respectivas, se evaluó calculando sus índices de homogeneidad y validez. Finalmente, las relaciones entre constructos de intereses, formas de vida y personalidad, se estudiaron mediante análisis correlacionales específicos.

\section{Resultados}

\section{Análisis de las características técnicas}

\section{Fiabilidad}

La fiabilidad o consistencia interna de cada una de las escalas RIASEC y de la Clave Profesional en su totalidad se obtuvo calculando los respectivos coeficientes ade Cronbach y 
Kuder-Richardson 20 (para ítems dicotomizados a efectos de su posterior comparación con la versión original del instrumento) como muestra la Tabla 1.

Tabla 1. Coeficientes de Fiabilidad de las escalas de la Clave Profesional

\begin{tabular}{lll}
\hline Escalas RIASEC & $\alpha$ de Cronbach & KR-20 \\
\hline (R) Realista & .723 & .717 \\
(I) Investigador & .850 & .857 \\
(A) Artístico & .821 & .759 \\
(S) Social & .735 & .660 \\
(E) Emprendedor & .802 & .784 \\
(C) Convencional & .724 & .739 \\
\hline La Clave Profesional & .802 & .819 \\
\hline
\end{tabular}

Como puede apreciarse, la fiabilidad global es elevada e incluso superior a los valores obtenidos en algunas escalas por Jones (1989). Además, se trata de valores adecuados conforme los que suelen encontrarse en este tipo de instrumentos, coeficientes test-retest[.7,.89] y $\alpha$ de Cronbach [.86, .91] (Jones, 1989; Holland, 1985, Jones \& Ward, 2002; Martínez-Vicente, 2003). En cuanto a los índices de fiabilidad de las escalas son muy satisfactorios, sobre todo si tenemos en cuenta el reducido $\mathrm{n}^{\circ}$ de ítems que componen cada tipo. Se confirma de este modo la gran consistencia de la adaptación realizada y en particular, de la escala Investigadora, por ser la más fiable.

\section{Validez Criterial}

La validez criterial, de gran utilidad diagnóstica, se calculó tomando como referencia el SDS (Holland, 1994) obteniéndose las intercorrelaciones mostradas en la Tabla 2. Anteriormente ya se había estudiado el ajuste entre las escalas de ambos instrumentos sin encontrar diferencias significativas entre sus escalas (Jones, Gorman \& Schroeder, 1989).

Tabla 2. Intercorrelaciones entre Clave Profesional y el SDS

\begin{tabular}{ll}
\hline Escalas RIASEC & $R_{X Y}$ \\
\hline (R) Realista & $.613^{* *}$ \\
(I) Investigador & $.821^{* *}$ \\
(A) Artístico & $.832 * *$ \\
(S) Social & $.711^{* *}$ \\
(E) Emprendedor & $.737 * *$ \\
(C) Convencional & $.715^{* *}$ \\
\hline$* * \mathrm{p}<.01$ &
\end{tabular}


Los índices de validez de todas las escalas de la Clave Profesional resultaron ser significativos y elevados, confirmándose de este modo el modelo RIASEC subyacente al instrumento y, particularmente, la buena definición de las escalas Artística e Investigadora.

Dado que la delimitación del perfil tipológico supone la clasificación de los sujetos en 6 tipos, además, se calculó el grado de acuerdo entre las clasificaciones de los alumnos en el primer tipo realizadas por la Clave Profesional y el SDS mediante el coeficiente Kappa (con valores entre -1 y 1 , indicando el cero aleatoriedad en la clasificación). Al obtener un valor de Kappa significativo $(.338, p<.05)$ se constata un grado de acuerdo moderado entre las clasificaciones de ambos instrumentos, semejante al obtenido por Jones (1989, 1990) (.37, $p<.05)$.

\section{Validez de Constructo}

La estructura hexagonal teóricamente subyacente a la Clave Profesional mostrada en la Figura 2, se verificó mediante el cálculo de la matriz de correlaciones entre sus escalas.. Las intercorrelaciones significativas más coherentes conforme el modelo de Holland fueron RC IS y SE seguidas de RI, AS, SC y EC. La consistencia que la configuración de tipos presenta en la muestra analizada, coincide en parte con las configuraciones más consistentes de Holland (RI, RC, AS, SE, CE) y algunas otras de consistencia intermedia (IS, SC). Respecto a la magnitud de las intercorrelaciones, ésta suele ser inferior a la teóricamente esperada e incluso suelen detectarse empíricamente algunas intercorrelaciones que no satisfacen la disposición hexagonal como es el caso de las relaciones RS, RE, IC, AE, que en la muestra actual no resultaron significativas (Castaño y otros, 1979; Jones, 1990; Fernández Eire, 1997; Holland, 1973, 1994; Lewis y Rivkin, 2000; Valls, 1999; Valls y MartínezVicente, 2001). 


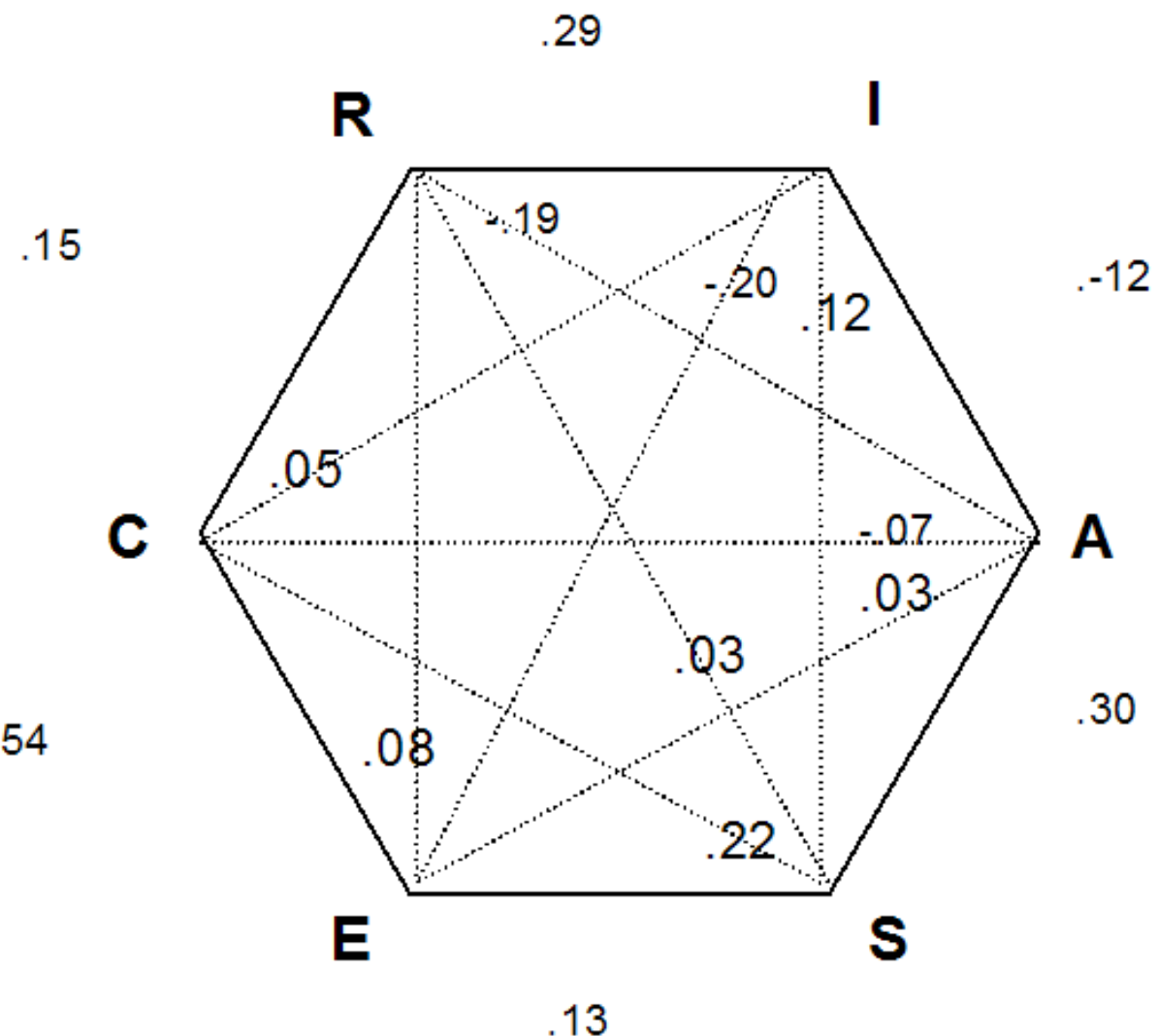

Figura 2. Correlaciones entre los tipos RIASEC (Clave Profesional)

No obstante, la validación de constructo es un proceso que implica diversos estudios para recoger múltiples indicios sobre la naturaleza del mismo, por ello se realizaron también diversos Análisis Factoriales sobre las dos primeras secciones de la Clave Profesional -¿Cómo me veo a mí mismo?, Ocupaciones preferidas- para comprobar si en el conjunto de ítems que constituían cada una de ellas (del 1 al 24 y del 25 al 60, respectivamente), aparecían claramente definidos los 6 tipos RIASEC. Como síntesis de estos análisis, es preciso señalar que se realizaron diversos análisis factoriales para cada una de las secciones con la obtención de las correspondientes matrices factoriales derivadas de las rotaciones ortogonales (Método Varimax) y oblícuas (método Oblimín Directo), pero en ambos casos se realizaron las interpretaciones de los factores sobre las ortogonales ya que las oblicuas aportaban muy poca información adicional. La selección de seis factores en ambas explicó el 58\% y el 51\% de la varianza total en la $1^{\mathrm{a}}$ y la $2^{\mathrm{a}}$ sección, respectivamente, siendo éstos porcentajes aceptables en comparación con los obtenidos en estudios similares, Holland y Gottfredson (1992). Además, las comunalidades de todos los ítems oscilaban en los intervalos $[.7, .4]$ y $[.7, .3]$ en la $1^{\mathrm{a}}$ y $2^{\mathrm{a}}$ sección respectivamente, por lo que la calidad de representación de estas soluciones factoriales era buena en ambos casos, en especial en los ítems 5(I), 9(A), 18(E), 1(R), 6(I), 
10(A), 13(S), 17(E), 39(R), 59(I), 35(I), 45(I) y 60(E). No obstante, tras el análisis de las matrices ortogonales, cabe realizar las siguientes observaciones:

- $\quad$ En la $1^{\text {a }}$ sección se aprecia claramente la existencia de 6 factores subyacentes conformes a los tipos de Holland, definidos fundamentalmente por los ítems señalados en la Tabla 3. El único ítem a eliminar, por su baja calidad de representación, (comunalidad=.34) y su saturación en un factor que teóricamente no le correspondería (el factor VI Convencional, en vez del factor IV, Realista), sería el ítem 4 -soy realista- y precisar la definición de los ítems 16 -soy amistoso, de confianza- 23 -valoro el éxito en el comercio- y 24 -soy ordenado-.

Tabla 3. Factores definidos en la $1^{\text {a }}$ Sección de la Clave Profesional

\begin{tabular}{ccccccc}
\hline Factores. & I & II & III & IV & V & VI \\
\hline Tipos & E & I & A & S & R & C \\
\hline Ítems & $17,18,19,20$ & $5,6,7,8$ & $9,10,11,12$ & $13,14,15$ & $1,2,3$ & 21,22
\end{tabular}

Nota. E= Emprendedor; I= Investigador; A= Artístico; $\mathrm{S}=$ Social; $\mathrm{R}=$ Realista; $\mathrm{C}=$ Convencional

- En la $2^{\mathrm{a}}$ sección, como puede verse en la Tabla 4, se encuentran muy bien definidos los factores I (I), III (S), IV (A) y V (R). Sin embargo, existe una fuerte confusión entre los tipos Convencional y Emprendedor reflejados en las ocupaciones atractivas, por lo que los factores II y VI no parecen claramente definidos. En consecuencia, esta $2^{a}$ sección parece peor definida desde el punto de vista tipológico, sugiriendo la necesidad de revisar las ocupaciones atractivas pertenecientes a los tipos Convencional y Emprendedor.

Tabla 4. Factores definidos en la $2^{\text {a }}$ Sección de la Clave Profesional

\begin{tabular}{lcccc} 
Factores. & I & III & IV & V \\
\hline Tipos & I & S & A & R \\
\hline Ítems & $59,35,45,47,57$, & $28,52,38,50$ & $56,42,32,30,54$, & $39,27,51,25$ \\
& 33 & & 44 &
\end{tabular}

Nota . $\mathrm{E}=$ Emprendedor; $\mathrm{I}=$ Investigador; $\mathrm{A}=$ Artístico; $\mathrm{S}=$ Social; $\mathrm{R}=$ Realista; $\mathrm{C}=$ Convencional

\section{Análisis de los ítems}

Al analizar el Índice de Homogeneidad se pudo apreciar que, en general, todos los ítems arrojaban valores de homogeneidad superiores al .1 salvo el ítem 26 (entrenador deportivo, escala social, $I H=.017)$ que, en consecuencia, debería ser eliminado. Este resultado, unido al de los análisis factoriales previos, permiten concluir que los ítems 4 -soy realista $(\mathrm{R})$, - 44 -diseñador gráfico(A) y 24 -soy ordenado(C), deberían ser revisados. 
Por otra parte, todos los Índices de Validez obtenidos al correlacionar cada ítem con la puntuación total de la escala a la que pertenecen en el SDS resultaron ser satisfactorios y superiores a .1 (excepto el ítem 26 -entrenador deportivo(S), $I V=.08$ ). Paralelamente, atendiendo a sus pequeños índices de validez, deberían mejorarse los ítems 4 (R) 44 (A) 24 (C) y 3 (R).

Las relaciones entre el modelo de Holland, el 16 PF y las Formas de Vida de Spranger

Dado que la Clave Profesional debería reflejar cierta tipología de personalidad conforme al supuesto de Holland sobre los intereses como expresión de personalidad y que, como antecedentes teóricos en la elaboración de este instrumento, aparecían las Formas de Vida de Spranger, se decidió estudiar la relación entre estas pruebas, analizando las intercorrelaciones entre las escalas del 16 PF, el Cuestionario de Ideas Personales y la Clave Profesional. En síntesis pueden ofrecerse las siguientes observaciones (todas ellas referidas a relaciones significativas, $r \geq .3, p<.05)$ :

Se constatan algunas diferencias en los tipos de Holland debidas al género. En particular, no aparecen relaciones significativas entre los factores de personalidad del 16PF y los tipos Convencional y Emprendedor en el caso de los varones pero sí en las mujeres, detectándose tanto relaciones positivas entre el tipo Emprendedor, la Extroversión e Independencia ( $r=.392, p<.01$ y $r=.349, p<.05$, respectivamente) y el tipo Convencional, el Control de la autoimagen y la Extroversión $(r=.280, p<.05$ y $r=.278, p<.01$, respectivamente $)$ como relaciones negativas entre el tipo Convencional y la Ansiedad ( $r=-.294, p<.05)$.

El tipo Realista, en los varones, se relaciona positivamente con la Independencia $(r=.335, p<.05)$. En las mujeres, este tipo se vincula negativamente a la Extroversión $(\mathrm{r}=-.266$, $p<.01)$ y positivamente a la Autosuficiencia $(r=.283, p<.05)$.

El tipo Investigador, en los varones, se relaciona positivamente con la Independencia $(r=.375, p<.05)$, negativamente con la Socialización $(r=-.392, p<.05)$, aspecto este último común a las mujeres que, en cambio, manifiestan relaciones positivas entre este tipo y la Ansiedad $(r=.286, p<.05)$. 
Sólo en el caso de las mujeres, el tipo Artístico se relaciona positivamente con la Extroversión $(r=.328, p<.05)$ y negativamente con la Socialización $(r=.-279, p<.05)$ y el tipo Social se relaciona positivamente con la Extroversión $(r=.394, p<.01)$.

En cuanto a las relaciones de los tipos RIASEC con las Formas de Vida de Spranger se observa que:

El Tipo Investigador parece estar muy bien definido al correlacionar positivamente con el estilo de vida Teórico y negativamente con el Económico, tanto en hombres $(r=.507$, $p<.01 ; r=-.353, p<.05)$ como en mujeres $(r=.376, p<.05 ; r=.377, p<.05)$.

El tipo Artístico no se relaciona significativamente con el estilo de vida Artístico. En el caso de las mujeres, este tipo se relaciona positivamente con valores económicos $(r=.377, p<.05)$. En los varones, se relaciona negativamente con los valores políticos $(r=-$ $.357, p<.05)$.

El tipo Social se relaciona -en las mujeres- con el estilo de vida Social ( $r=.347$, $p<.05)$, mientras que en los hombres se vincula en mayor grado a valores religiosos $(r=.421, p<.05)$.

El tipo Emprendedor se relaciona positivamente con valores políticos y artísticos en las mujeres ( $r=.330, p<.05$ y $r=.334, p<.05$ respectivamente). En los hombres, se relaciona negativamente con los valores teóricos $(r=-.350, \mathrm{p}<.05)$.

El tipo Convencional no se relaciona significativamente con los valores en los hombres. Sin embargo, en las mujeres se relaciona positivamente con valores políticos $(r=.366, \mathrm{p}<.01)$ y negativamente con valores económicos $(r=-.284, \mathrm{p}<.05)$.

\section{Discusión y conclusiones}

Los resultados obtenidos permiten afirmar que la Clave Profesional es un instrumento de Diagnóstico y Orientación Profesional conforme al modelo tipológico de Holland, válido en muestras españolas semejantes a la considerada en este estudio. Muy fiable tanto 
globalmente (.8014), como en cada una de sus escalas -especialmente la Investigadora- su validez criterial es muy adecuada -sobre todo en las escalas Artística e Investigadora- y además realiza clasificaciones significativamente semejantes a las del SDS. Su estructura hexagonal se confirma globalmente, detectándose los seis factores de Holland. Ahora bien, la $1^{a}$ sección está mejor definida que la $2^{\mathrm{a}}$ en la cuál habría que reformular los ítems de ocupaciones que definen los tipos Emprendedor y Convencional. Por su parte, las intercorrelaciones entre escalas RIASEC siguen tendencias semejantes a las obtenidas en los estudios de Holland (1985, 1994) y de otros autores españoles (Fernández Eire, 1997; Martínez Vicente y Valls, 1999, etc.), de modo que emergen algunas correlaciones por su coherencia teórica (RI, AS, SC, EC), pero también surgen pequeños desajustes en las relaciones entre tipos, en especial en la escala Convencional, que parece siempre la peor definida incluso cuando este tipo de instrumentos se aplica a través de internet (Levinson, Zeman y Ohler, 2002). Tras el análisis de ítems puede afirmarse que la Clave Profesional presenta índices de homogeneidad y validez satisfactorios. No obstante, se sugiere eliminar o reformular los ítems 4, 24, 26 y 44 y estudiar los ítems 3, 16, 23, 37, 40 y 49 para definirlos nuevamente y apreciar si sus modificaciones afectan la calidad técnica del instrumento.

Paralelamente, se han confirmado ciertas relaciones con otros constructos como los valores y modelos de personalidad como el del 16PF, que es preciso matizar. Respecto a las relaciones entre la Clave Profesional y al $16 P F$, tal como afirmaban Holland \& Gottfredson (1992) no parece que estos dos tipos de pruebas sean intercambiables, aunque parece evidente la existencia de relaciones significativas entre los constructos de intereses y personalidad. Se confirman las relaciones entre las escalas RIASEC y la dimensión de Extroversión (Goh \& Leong, 1993), en particular, relaciones positivas con los tipos Social y Emprendedor (Tokar \& Swanson, 1995) pero otras relaciones no resultan tan claras como las relaciones negativas entre el tipo Emprendedor y el neuroticismo, el tipo investigador y la extroversión, entre el tipo Emprendedor y la extroversión -ésta última sólo confirmada en el caso de las mujeres-.

Tal vez el modelo tipológico de Holland suponga un enfoque diferente de personalidad, cuestión a investigar con otros modelos de personalidad como el de los Cinco Grandes Factores (Five Factor Model) cuyo lenguaje común facilitaría la base para estudiar las relaciones entre ambos dominios. 
Como era de esperar, encontramos diversas relaciones entre el modelo de Holland y las Formas de Vida de Spranger, algunas muy coherentes, como la vinculación positiva del tipo Investigador con los valores teóricos y negativa con los económicos, las del tipo Social con los valores sociales -especialmente en las mujeres- y las del tipo Emprendedor con valores políticos. No obstante, otros resultados -la ausencia de relación entre el tipo Artístico y los valores artísticos- apuntan la necesidad de posteriores investigaciones para profundizar en las relaciones entre ambos constructos.

En definitiva, se aporta un instrumento, la Clave Profesional, válido en nuestro contexto cultural y de gran utilidad por su brevedad y carácter motivador, en el Diagnóstico de estilos de vida y la Orientación profesional. Este tipo de instrumentos resultan ser eficaces, eficientes y estimuladores de la exploración de la carrera, especialmente cuando tras su aplicación, se sugieren formas específicas para indagar oportunidades profesionales (Jones, 2000). No obstante, se recomiendan futuros estudios en nuestro contexto cultural, para analizar su validez convergente y divergente, análisis factoriales confirmatorios etc. pertinentes para mejorar este instrumento así como para estudiar el impacto que este tipo de instrumentos puede tener tanto en los estudiantes que son menos "activos" en su exploración profesional como aquellos cuyas aspiraciones profesionales $-\mathrm{y}$ educativas- son tremendamente diversas.

Finalmente, este tipo de estudios empíricos permiten reelaborar una estructura teórica más coherente con la autorregulación individual hacia el conocimiento del "fermento interior" de la personalidad, que está permanentemente bullendo entre las necesidades, los intereses, las capacidades, los tipos ambientales de Holland y las formas de vida de Spranger. La autorregulación cognitiva en los procesos de elección profesional permite conformar un "estilo de vida" o concreción conductual coherente con los valores que cada persona asume y estos presupuestos son imprescindibles para adoptar decisiones y autorregular el desarrollo personal. 


\section{Referencias}

Álvarez-Rojo, V. (1991). Tengo que decidirme. Sevilla: Alfar.

Allen, J. \& Robbins, S. (2008). Prediction of college major persistence based on vocational interests and first-year academic performance. Research in Higher Education, 49, 6279. doi:10.1007/s11162-007-9064-5

Allen, J. \& Robbins, s. (2010). Effects of interest-major congruence, motivation, and academic performance on timely degree attainment. Journal of Counseling Psychology, 57, 23-35. http://dx.doi.org/10.1037/a0019085

Allport, G.W. \& Vernon, P.E. (1931). A tests for personal values. Journal of Abnormal and Social Psychology, 26, 231-248. http://dx.doi.org/10.1037/h0073233

Allport, G.W., Vernon, P.E. \& Liendzey, G. (1951). Study of values. Cambridge: Cambridge University Press.

Caprara, G.V., Barbaranelli, C. y Borgogni, L. (2001). BFQ Cuestionario “Big Five” (3ª ed.). Madrid: TEA.

Castaño, C. (1977). Uso del Temario Vocacional de García Yagüe en la orientación de estudios. Madrid: Miñón.

Castaño, C., López Navarro, J.A. y Domínguez, A. (1979). Verificación en la población española de la estructura de intereses de la hipótesis tipológica de Holland. Revista de psicología general y aplicada, 160-161, 943-946.

Cattell, R.B. (1975). Cuestionario de personalidad 16 PF. Madrid: TEA.

Corominas, E, Álvarez González, M. y Bisquerra, R. (1999). Programa Autoaplicado de Orientación Profesional, (POPA). Barcelona: Servicio de publicaciones de la Generalidad de Cataluña.

Corominas, E, Álvarez González, M. y Bisquerra, R. (2009). GR: Herramienta de autoaplicación para la orientación académica y profesional. Recuperado de http:// http://orientacion.educaweb.com/es/presentacion.

Delgado, J.A. (1995). Toma la iniciativa. Programa de Orientación Vocacional para el primer grado de la ESO. Granada: Universidad de Granada.

Fernández Eire, L. (1997). Eleccións de Carreiras e Profesións: Adaptación Self-Direted Search, forma regular (1994) a adolescetes galegos (Tesis doctoral). Santiago de 
Compostela: Servicio de Publicaciones e Intercambio Científico de la Universidad de Santiago de Compostela.

García-Yagüe, J. (1957). Cuestionario de ideas personales. Madrid: CSIC.

García-Yagüe, J y Lázaro, A. (1971). Condicionamientos ambientales de la personalidad. Madrid: Magisterio español.

Goh, D.S. \& Leong, F.T.L. (1993). The relationship between Holland's Theory of Vocational Interest and Eysenck's Model of Personality. Personality and individual differences, 15, 555-562. doi: 10.1016/0191-8869(93)90339-5

Gottfredson, G.D. (1999). John L. Holland's Contributions to Vocational Psychology: A Review and Evaluation. Journal of Vocational Behavior, 55(1), 14-40. doi: 10.1006/jvbe.1999.1695

Gottfredson, G.D. \& Holland, J.L. (1991). Position Classification Inventory: Professional Manual. Odessa, FL.: Psychological Assessment Resources.

Gottfredson, G. D., \& Johnstun, M. (2009). John Holland's contributions: A theory-riddent approach to career assistance. Career Development Quarterly, 58, 99-107. doi: 10.1002/j.2161-0045.2009.tb00050.x

Holland, J.L. (1959). A theory of vocational choice. Journal of Counseling Psychology, 6, 3545. http://dx.doi.org/10.1037/h0025350

Holland, J.L. (1962). Some explorations of a Theory of Vocational Choice: 1. One and two years longitudinal studies (Monograph). Psychological Monographs, 76, 26, Whole No. 545).

Holland, J.L. (1968). Explorations of a Theory of Vocational Choice: VI. A longitudinal study using sample of typical college students. Journal of Applied Psychology, 52(1, Pt.2). 1-37. http://dx.doi.org/10.1037/h0025350

Holland, J.L. (1979). The Self-Directed Search Professional Manual. Palo Alto, CA: Consulting Psychologist Press.

Holland, J.L. (1985). Making vocational Choices: a Theory of Vocational Personalities and Work Environments ( $2^{\text {nd }}$ ed.). Englewood Cliffs, NJ.: Prentice-Hall.

Holland, J.L. (1994). The Self-Directed Search. Odessa, FL: Psychological Assessment Resources.

Holland, J.L. (1997). Making Vocational Choices: a Theory of Vocational Personalities and Work Environments. ( $3^{\text {rd }}$ ed.). Odessa, FL: Psychological Assessment Resources. 
Holland, J.L.. \& Gottfredson, G.D. (1976). Using a typology of persons and environments to explain careers: some extensions and clarifications. Counseling Psychologist, 6, 20-29. doi: $10.1177 / 001100007600600306$

Holland, J.L. \& Gottfredson, G.D. (1978). New edition of the Self-Directed Search. JSAS Catalog of Selected Documents in Psychology. 8, 73, ( $\left.\mathrm{n}^{\circ} 1740\right)$.

Holland, J.L. \& Gottfredson, G.D. (1992). Studies of the Hexagonal Model: an evaluation or the perils of stalking the perfect hexagon. Journal of Vocational Behavior, 40, 158170. doi:10.1016/0001-8791(92)90063-6

Holland, J.L. \& Nichols, R.C. (1964). Explorations of a Theory of Vocational Choice: III. A longitudinal study of change in a major field of study. Personnel and Guidance Journal, 11, 235-242. doi: 10.1002/j.2164-4918.1964.tb02667.x

Holland, J.L., Sorensen, A.B., Clark, J.P., Nafzinger, D.H. \& Blum, Z.D. (1973). Applying and occupational classification to a representative sample of work histories. Journal of Applied Psychology, 58, p. 34-41.

Holland, J.L. \& Whitney, D.R., Cole, N.S., Richards, J.M. (1969). An empirical occupational classification derived from a theory of personality and intended for practice and research (ACT Research Report $\mathrm{n}^{\circ}$ 29). Iowa City: The American College Testing Program.

Jones, L.K. (1987). The Career Key. Chicago, J.G.: Ferguson.

Jones, L.K., Gorman, S. Schroeder, C.G. (1989). A comparison between the SDS and the Career Key among career undecide college students. The Career Development Quarterly, 37, 334-344. doi: 10.1002/j.2161-0045.1989.tb00673.x

Jones, L. K. (1990). The Career Key: An investigation of the reliability and validity of its scales and its helpfulness to college students. Measurement and Evaluation in Counseling and Development, 23, 67-76.

Jones, L.K. (1993). Two career guidance instruments: their helpfulness to students and effect on students's career. School Counselor, 40(3), 191-204.

Jones, L.K. Sheffield, D. \& Joyner, B. (2000). Comparing the effects of the Career Key with Self-Directed Search and Job-OE among eig-grades students. Professional School Counseling, 3(4), 238-247.

Jones, L. K., \& Ward, M. C. (2002). Equivalence of three versions of the Career Key among high school students. Hood River, OR: Career Key. 
Kristof-Brown, A.L., Zimmerman, R.D. \& Johnson, E.C. (2005). Consequences of individuals' fit at work: A meta-analysis of person-job, person-organization, persongroup, and person-supervisor fit. Personnel Psychology, 58, 281-342.

Lázaro, A. y Mudarra, M.J. (2005). Las competencias del orientador en la movilidad $\begin{array}{llll}\text { profesional. } & \text { Qurriculum, } & 18, & 175-191 .\end{array}$ http://webpages.ull.es/users/revistaq/ANTERIORES/numero18/lazaro.pdfLevinson, E.M., Zeman, H.L. \& Ohler, D.L. (2002). A critical evaluation of the web-based version of the Career Key. The Career Development Quarterly, 51, 26-35. doi: 0.1002/j.2161-0045.2002.tb00589.x

Lewis, P.H. \& Rivkin, D. (2000). O*Net Interest Profiler v 3.0: user's guide. Raleigh, NC: US Department of Labor and Employment and Training Administration.

Martínez-Vicente, J.M. y Valls Fernández, F. (1999). Estudio preliminar para la adaptación del programa autoaplicable de elección vocacional Self-Directed Search de John L. Holland, Revista Española de Orientación y Psicopedagogía, 10(18), 353-368. Recuperado de http://www.uned.es/reop/pdfs/1999/10-18-2---353Jose\%20Manuel\%20Martinez.PDF

Martínez-Vicente, J.M. (2001). Estudio Adaptación del Self-Directed Search Form R (SDS) y del Position Classification Inventory (PCI) a la población española. Almería: Servicio de publicaciones de la Universidad de Almería. Recuperado de http://www.investigacion-psicopedagogica.org/revista/new/ContadorArticulo.php?166

Martínez-Vicente, J.M. y Valls Fernández, F. (2001). Validez transcultural del modelo hexagonal de personalidad propuesto en la teoría tipológica de elección vocacional de John Holland. Revista de Psicología General y Aplicada, 54(4), 577-586.

Martínez-Vicente, J.M., Valls, F. y Álvarez, J. (2006). La personalidad y conducta vocacional. En F. Rivas (Ed.), Asesoramiento vocacional: Teoría, práctica e instrumentación (pp.279-311). Barcelona: Ariel.

Martínez-Vicente, J.M. y Valls Fernández, F. (2006). La elección vocacional y la planificación de la carrera. Adaptación española del Self-Directed Search (SDS-R) de Holland. Psicothema, 18, 1, 117-122.

Martínez-Vicente, J.M. (2007). El asesoramiento vocacional y profesional a través del SelfDirected Search (SDS). Electronic Journal of Research in Educational Psychology, 
5(11),233-258. Recuperado de http://www.investigacionpsicopedagogica.org/revista/new/ContadorArticulo.php?166

Martínez-Vicente, J.M. y Valls Fernández, F. (2008). Aplicación de la Teoría de Holland a la clasificación de ocupaciones: Adaptación del Inventario de Clasificación de Ocupaciones (ICO). Revista Mexicana de Psicología 25(1), 151-164.

Mudarra, M.J. (2003). Propuesta de un Sistema de Autoevaluación de Áreas Profesionales para la Orientación de la Carrera. (Tesis doctoral). Madrid: UNED.

Mudarra, M.J. (2007). El Sistema de Autoevaluación de Áreas Profesionales: un instrumento de diagnóstico y Orientación profesional. Educación XX1, 10, 195-213. doi: http://dx.doi.org/10.5944/educxx1.1.10.303

Porter, S.R. \& Umbach, P.D. (2006). College major choice: An analysis of personenvironment fit. Research in Higher Education, 47, 429-449. doi: 10.1007/s11162005-9002-3

Raymond Ting, S. (2009). A preliminary study on the effectiveness of the chinese Career Key online. The Career Development Querterly, 58- 77-81. doi: 10.1002/j.21610045.2009.tb00176.x

Repetto, E. (1999). Tu futuro profesional. Madrid: CEPE.

Romero, S. (1997). De gira hacia el trabajo: Programa A.D.V.P. de Desarrollo de Carrera. Comunidad Educativa, 240, 30-33.

Romero, S. (2000). De gira hacia el trabajo. Guía del profesorado. Granada: Aljibe.

Rivas, F. y López, M.L. (2003). Sistema de Autoayuda y Asesoramiento Vocacional Revisado $(S A A V-R)$. Madrid: EOS.

Santana Vega, L., Feliciano, L. y Cruz, A. (2010). El Programa de Orientación Educativa y Sociolaboral: Un instrumento para facilitar la toma de decisiones en Educación Secundaria. Revista de Educación, 351, pp.73-105. Recuperado de http://www.revistaeducacion.mec.es/re351/re351_04.pdf

Savickas, M.L. \& Gottfredson, G.D. (1999). Holland's Theory (1959-1999): 40 years of research and application. Journal of Vocational Behavior, 55(1), 1-5. doi: 10.1006/jvbe.1999.1694

Savickas, M.L., Taber, B. \& Spokane, A. (2002). Convergent and discriminant validity of five interest inventories. Journal of Vocational Behavior, 61(1), 139-184. doi: 10.1006/jvbe.2002.1878 
Skinner, Q. (1988). El retorno de la gran Teoría en las Ciencias Humanas. Madrid: Alianza.

Smart, J.C. (2010). Differential patterns of change and stability in student learning outcomes in Holland's academic environments: the role of environmental consistency. Research in Higher Education, 51, 469-482. doi:10.1007/s11162-010-9163-6

Spranger, E. (1935). Formas de vida. Psicología y ética de la personalidad (1 $\left.{ }^{\mathrm{a}} \mathrm{ed}\right)$. Madrid: Revista de Occidente.

Spranger, E. (1953). Test formas de vida. Madrid: CSIC.

Tokar, D.M. \& Swanson, J.L. (1995). Evaluation of the correspondence between Holland's Personality Typology and the Five-Factor Model of Personality. Journal of vocational behavior, 46, 89-108. doi: 10.1006/jvbe.1995.1006

Tracey,T.J.C. \& Robbins, S.B. (2006). The interest-major congruence and college success relation: a longitudinal study. Journal of Vocational Behavior 69, 64-89. doi: 10.1016/j.jvb.2005.11.003

Valls, F. (1996). Programa Autoaplicable de Orientación Vocacional. Almería: Universidad de Almería.

Valls, F. (2007). Inteligencia emocional y asesoramiento vocacional: usos y abusos. Electronic Journal of Research in Educational Psychology, 5(11), 179-200. Recuperado de http://www.investigacion-psicopedagogica.org/revista/new/ContadorArticulo.php?162 